\title{
On a vector potential formulation for 3D electromechanical finite element analysis
}

\author{
A. S. Semenov ${ }^{*, \dagger}$, H. Kessler, A. Liskowsky and H. Balke \\ Inst. für Festkörpermechanik, TU Dresden, Mommsenstr. 13, 01069 Dresden, Germany
}

\begin{abstract}
SUMMARY
A vector potential for the electric induction is applied to static three-dimensional fully coupled electromechanical problems. A Coulomb gauge condition imposed on the electric vector potential improves the convergence behaviour of nonlinear problems, and in combination with a discrete set of Dirichlet boundary conditions, it can enforce unique vector potential solutions. Based on a spectral analysis of the stiffness matrix, the Coulomb gauge is compared with other gauge conditions. A penalized version of the weak vector potential formulation with Coulomb gauge is proposed and tested on some numerical examples in electrostatics, piezoelectricity and ferroelectricity. Copyright (C) 2005 John Wiley \& Sons, Ltd.
\end{abstract}

KEY WORDS: electric vector potential; three-dimensional finite element analysis; gauge condition; piezoelectricity; ferroelectricity

\section{INTRODUCTION}

The Maxwell equations to be satisfied in electrostatics or static electromechanical problems with a vanishing volume density of free charges are [1]:

$$
\nabla \times \mathbf{E}=\mathbf{0}
$$

and

$$
\nabla \cdot \mathbf{D}=0
$$

\footnotetext{
${ }^{*}$ Correspondence to: A. S. Semenov, Inst. für Festkörpermechanik, TU Dresden, Mommsenstr. 13, 01069 Dresden, Germany.

†E-mail: pantocrator@inbox.ru

Contract/grant sponsor: German Research Foundation (DFG)
}

Copyright (c) 2005 John Wiley \& Sons, Ltd.

Received 8 September 2004

Revised 24 May 2005

Accepted 7 June 2005 
Corresponding to (1)-(2), there are two formulations of the field problem with different choices of the primary electric variables:

(i) The electric field intensity $\mathbf{E}$ is expressed by the scalar electric potential $\phi$, and (1) is satisfied automatically

$$
\mathbf{E}=-\nabla \phi \quad \rightarrow \quad \nabla \times \mathbf{E}=\mathbf{0}
$$

(ii) The electric flux $\mathbf{D}$ is expressed by a vector potential $\psi$, and (2) is satisfied automatically

$$
\mathbf{D}=-\nabla \times \boldsymbol{\psi} \rightarrow \nabla \cdot \mathbf{D}=0
$$

Equations (3) and (4) find their counterparts in finite element (FE) analysis of coupled electromechanical problems with corresponding choices of the electric nodal variables using $\phi$ in the standard scalar potential formulation [2] and $\psi$ in the recently offered vector potential formulation [3]. In coupled electromechanical FE formulations the electric vector potential leads to a positive definite stiffness matrix, in contrast to formulations based on the scalar electric potential. Therefore, solutions of boundary value problems using scalar potential formulation lie on a saddle point, while solutions for the vector potential formulation exist at a global minimum in the space of the nodal degrees of freedom [3]. This difference favours the electric vector potential especially for the solution of nonlinear electromechanical problems. Vector potential formulation is superior for nonlinear problems also due to ability of ferroelectrics to accumulate 'plastic' polarization and not 'plastic' electric field, and hence the formulation that derives polarization from nodal degrees of freedom is more readily implemented for ferroelectrics.

The examples in Reference [3] illustrating the vector potential were two-dimensional. Threedimensional vector potential solutions of the equilibrium conditions are - in contrast to the two-dimensional case - nonunique, leading to an ill-conditioned (rank-deficient) stiffness matrix. Uniqueness can be enforced by imposing an additional gauge condition and appropriate boundary conditions. In our FE implementation, the Coulomb gauge known from magnetostatics [1] will be satisfied employing the penalty function method [4]. A brief comparison will be made to the electric versions of some other gauge conditions, adapted from magnetostatics and eddy current problems [5-9]. The influence of the penalty value on the accuracy of the solution will be investigated for some examples of homogeneous and heterogeneous materials, and some practical recommendations will be given.

In the next three sections, we first discuss the dielectric case, followed by consideration of coupled electromechanical problems in piezoelectric solids and by presentation of numerical examples.

\section{THE VECTOR POTENTIAL IN ELECTROSTATICS}

\subsection{Boundary value problem}

The field equations (1)-(2) in the domain $V$ have to be complemented by boundary conditions on the outer surface of $V$ composed of $S=S_{D} \cup S_{E}\left(S_{D} \cap S_{E}=\emptyset\right)$ :

$$
\mathbf{n} \cdot \mathbf{D}=D_{n} \quad \text { on } S_{D}
$$




$$
\mathbf{n} \times \mathbf{E}=\mathbf{E}_{S} \quad \text { on } S_{E}
$$

where $\mathbf{n}$ represents the normal unit vector on $S ; D_{n}$ is the normal electric displacement outside of $V$ reduced by the surface density of free charges; $\mathbf{E}_{S}$ denotes a given vector in the tangential plane of $S_{E}$, which can be expressed by the scalar potential $\phi$ as $\mathbf{E}_{S}=-\mathbf{n} \times \nabla \phi$.

In the presence of an internal interface $S_{12}$ between two sub-domains $V_{1}$ and $V_{2}$ of $V$ with different material properties, continuity conditions have to be enforced:

$$
\begin{aligned}
\mathbf{n} \cdot \mathbf{D}_{1} & =\mathbf{n} \cdot \mathbf{D}_{2} \\
\mathbf{n} \times \mathbf{E}_{1} & =\mathbf{n} \times \mathbf{E}_{2}
\end{aligned} \quad \text { on } S_{12}
$$

where the indices 1 and 2 refer to $V_{1}$ and $V_{2}$, and $\mathbf{n}$ denotes the interface normal. In addition to the field equations and boundary conditions, a complete formulation of the boundary value problem requires a constitutive relation between $\mathbf{E}$ and $\mathbf{D}$. For linear materials without remanent polarization, the dielectric constitutive relation is given by

$$
\mathbf{E}=\boldsymbol{\beta} \cdot \mathbf{D}
$$

where $\boldsymbol{\beta}$ represents a load-independent second rank tensor of dielectric moduli. The field equations (1) $-(2)$, boundary conditions $(5)-(7)$ and constitutive relation (8) ensure uniqueness of the field quantities $\mathbf{D}$ and $\mathbf{E}$.

The boundary value problem (1)-(2), (5)-(8) can be reformulated in terms of the electric vector potential $\psi$ defined by $(4)_{1}$ :

$$
\begin{aligned}
\nabla \times[\boldsymbol{\beta} \cdot(\nabla \times \boldsymbol{\psi})] & =\mathbf{0} \quad \text { in } V \\
\mathbf{n} \cdot(\nabla \times \boldsymbol{\psi}) & =-D_{n} \quad \text { on } S_{D} \\
\mathbf{n} \times \boldsymbol{\beta} \cdot(\nabla \times \boldsymbol{\psi}) & =-\mathbf{E}_{S} \quad \text { on } S_{E} \\
\mathbf{n} \cdot\left(\nabla \times \boldsymbol{\psi}_{1}\right) & =\mathbf{n} \cdot\left(\nabla \times \boldsymbol{\psi}_{2}\right) \quad \text { on } S_{12} \\
\mathbf{n} \times \boldsymbol{\beta}_{1} \cdot\left(\nabla \times \boldsymbol{\psi}_{1}\right) & =\mathbf{n} \times \boldsymbol{\beta}_{2} \cdot\left(\nabla \times \boldsymbol{\psi}_{2}\right) \quad \text { on } S_{12}
\end{aligned}
$$

\subsection{Uniqueness of the electric vector potential}

The solution $\psi$ of the boundary value problem (9) involving the 'curl-curl' operator is nonunique since the equations are invariant under the transformation $\psi \rightarrow \boldsymbol{\psi}^{\prime}=\boldsymbol{\psi}+\nabla \chi$, where $\chi(\mathbf{r})$ is an arbitrary continuous and differentiable function. The electric vector potential $\psi$ is arbitrary in the sense that a gradient can be added without changing $\mathbf{D}$ and $\mathbf{E}$, as the curl of any gradient is zero. Contrary to the boundary value problem (1)-(2), (5)-(8) for the physical fields $\mathbf{D}$ and $\mathbf{E}$, one can obtain an infinite number of solutions to the boundary value problem (9). This fact leads in the context of the FE method to ill-conditioned (rank-deficient) stiffness matrices. An unique vector potential solution can be enforced by imposing additional gauge conditions.

Similar observations are made for the magnetic vector potential in magnetostatics and eddy current problems. A number of different gauging strategies have been proposed and applied in 
computations: the Coulomb gauge imposed in the domain of analysis $V$ [5-8] or on the outer boundary $S$ [10], the two-component gauge [11], the incomplete gauge [9], the three-field formulation [12] and the tree-gauge method [13]. An alternative approach based on the direct elimination of the degrees of freedom corresponding to vanishing eigenvalues of the stiffness matrix was suggested by McMeeking and Baumgarten [14] in application to ferroelectric problems. An ungauged formulation used in Reference [15] ensures uniqueness of the field quantities $\mathbf{D}$ and $\mathbf{E}$, but not of the vector potential $\psi$.

The Coulomb gauge condition is used in the present study to ensure the uniqueness of the electric vector potential. According to Helmholtz' theorem [16], a vector field is uniquely determined by specifying both its curl and divergence supplemented by appropriate boundary conditions. With $(4)_{1}$, the electric induction $\mathbf{D}(\mathbf{r})$ - taking the view it is given as solution of the boundary value problem - determines only the curl of $\psi$. Therefore, an additional constraint on the divergence of $\psi$ may be imposed without changing the physically relevant fields $\mathbf{D}(\mathbf{r})$ and $\mathbf{E}(\mathbf{r})$. It is reasonable to simplify Equation $(9)_{1}$ by the choice of $\nabla \cdot \psi$. Equation $(9)_{1}$ can be rewritten in the case of isotropic homogeneous materials as

$$
\nabla \times(\nabla \times \boldsymbol{\psi})=\nabla(\nabla \cdot \boldsymbol{\psi})-\Delta \boldsymbol{\psi}=\mathbf{0}
$$

The Coulomb gauge condition

$$
\nabla \cdot \psi=0
$$

reduces (10) to the Laplace's equation

$$
\Delta \psi=0
$$

This simplification holds only for isotropic homogeneous materials. More complex equations are obtained in the general case of arbitrary materials, but the Coulomb gauge condition (11) ensures the uniqueness of $\psi$ in all cases.

The solution of the vector potential problem in a bounded domain requires additional appropriate boundary conditions to ensure uniqueness. A possible set of conditions is given in Reference [5] by

$$
\begin{aligned}
\mathbf{n} \times \boldsymbol{\psi}=\mathbf{P} & \text { on } S_{D} \\
\mathbf{n} \cdot \boldsymbol{\psi}=0 & \text { on } S_{E} \\
\boldsymbol{\psi}_{1}=\boldsymbol{\psi}_{2} & \text { on } S_{12}
\end{aligned}
$$

where $\mathbf{P}$ is an arbitrary vector function satisfying the condition $\mathbf{n} \cdot \mathbf{P}=0$.

A gauge condition is required only in three dimensions. In two dimensions with one nonvanishing component of the vector potential, $\psi_{z}(x, y)$, the Coulomb gauge condition (11) is satisfied automatically. We emphasize further that gauging is not required to get a solution for the field problem (1)-(2), (5)-(8). However, it effects the solution process of the field problem (9) for $\psi$ and, in particular, its convergence and accuracy of the results.

\subsection{Boundary value problem with Coulomb gauge}

The formulation of the boundary value problem in terms of the vector potential, which yields an unique solution, combines Equations (9) and the gauge conditions (11), (13) 
as follows:

$$
\begin{aligned}
\nabla \times[\boldsymbol{\beta} \cdot(\nabla \times \boldsymbol{\psi})] & =\mathbf{0} \quad \text { in } V \\
\nabla \cdot \boldsymbol{\psi} & =0 \quad \text { in } V \\
\mathbf{n} \times \boldsymbol{\psi} & =\mathbf{P} \quad \text { on } S_{D} \\
\mathbf{n} \times \boldsymbol{\beta} \cdot(\nabla \times \boldsymbol{\psi}) & =-\mathbf{E}_{S} \quad \text { on } S_{E} \\
\mathbf{n} \cdot \boldsymbol{\psi} & =0 \quad \text { on } S_{E} \\
\mathbf{n} \times \boldsymbol{\beta}_{1} \cdot\left(\nabla \times \boldsymbol{\psi}_{1}\right) & =\mathbf{n} \times \boldsymbol{\beta}_{2} \cdot\left(\nabla \times \boldsymbol{\psi}_{2}\right) \quad \text { on } S_{12} \\
\boldsymbol{\psi}_{1} & =\boldsymbol{\psi}_{2} \quad \text { on } S_{12}
\end{aligned}
$$

The boundary condition $(9)_{2}$ has been replaced in (14) by a Dirichlet type condition $\mathbf{n} \times\left.\boldsymbol{\psi}\right|_{S_{D}}=\mathbf{P}$ [5]. $\mathbf{P}$ is an otherwise arbitrary vector field defined on the surface $S_{D}$, which is required to satisfy the auxiliary condition $\tilde{\nabla} \cdot \mathbf{P}=D_{n}$ on $S_{D}$, where $\tilde{\nabla}$ denotes the surface part of the nabla-operator $\nabla=\tilde{\nabla}+\mathbf{n} \partial_{n}$. The proof that $\tilde{\nabla} \cdot \mathbf{P}=D_{n}$ guarantees indeed recovery of the original boundary condition $(9)_{2}$ employs Weingarten's equation $\tilde{\nabla} \times \mathbf{n}=\mathbf{0}$ [17]: Then it can be shown on $S_{D}$ that

$$
\mathbf{n} \cdot(\nabla \times \boldsymbol{\psi})=\mathbf{n} \cdot(\tilde{\nabla} \times \boldsymbol{\psi})=\mathbf{n} \cdot(\tilde{\nabla} \times \boldsymbol{\psi})-\boldsymbol{\psi} \cdot(\tilde{\nabla} \times \mathbf{n})=-\tilde{\nabla} \cdot(\mathbf{n} \times \boldsymbol{\psi})=-\tilde{\nabla} \cdot \mathbf{P}=-D_{n}
$$

In the case of isotropic homogeneous materials, the Equation (14) $)_{1,2}$ convert to Laplace's equation (12). In the general case, special procedures are required. There are different methods to ensure an exact or approximate satisfaction of constraint equations, as for example the method of Lagrange multipliers or the penalty function method. In contrast to the method of Lagrange multipliers, the penalty function method does not introduce additional unknowns. However, the selection of suitable penalty values is not a trivial task. Application of the penalty method to enforce the Coulomb gauge condition leads to a modified version of the field equations $(14)_{1,2}$ :

$$
\nabla \times[\boldsymbol{\beta} \cdot(\nabla \times \boldsymbol{\psi})]-\nabla(\alpha \nabla \cdot \boldsymbol{\psi})=\mathbf{0} \text { in } V
$$

where $\alpha$ is an empirically chosen penalty factor. The divergence of (16) yields Laplace's equation for $\alpha \nabla \cdot \psi$ :

$$
\Delta(\alpha \nabla \cdot \psi)=0 \text { in } V
$$

Vanishing solutions of Equation (17), $\alpha \nabla \cdot \psi \equiv 0$, recover the original gauge condition $(14)_{2}$ and the original field equation $(14)_{1}$. This is possible, for example, if homogeneous Dirichlet or Neumann boundary conditions could be enforced on $\alpha \nabla \cdot \psi$ along $S$. On $S_{E}$, Equation (14) replaces Neumann boundary condition for $\alpha \nabla \cdot \psi$ [5]. Thus, a Dirichlet boundary condition $\alpha \nabla \cdot \psi=0$ must be given only along $S_{D}$ in order to satisfy the Coulomb gauge condition. The continuity condition on the interface $S_{12}$ for Equation (17) is given by $\alpha_{1} \nabla \cdot \psi_{1}=\alpha_{2} \nabla \cdot \psi_{2}$. In summary, the following boundary value problem with penalized Coulomb gauge has to 
be solved:

$$
\begin{aligned}
\nabla \times[\boldsymbol{\beta} \cdot(\nabla \times \boldsymbol{\psi})]-\nabla(\alpha \nabla \cdot \boldsymbol{\psi}) & =\mathbf{0} \quad \text { in } V \\
\mathbf{n} \times \boldsymbol{\psi} & =\mathbf{P} \quad \text { on } S_{D} \\
\alpha \nabla \cdot \boldsymbol{\psi} & =0 \quad \text { on } S_{D} \\
\mathbf{n} \times \boldsymbol{\beta} \cdot(\nabla \times \boldsymbol{\psi}) & =-\mathbf{E}_{S} \quad \text { on } S_{E} \\
\mathbf{n} \cdot \boldsymbol{\psi} & =0 \quad \text { on } S_{E} \\
\mathbf{n} \times \boldsymbol{\beta}_{1} \cdot\left(\nabla \times \boldsymbol{\psi}_{1}\right) & =\mathbf{n} \times \boldsymbol{\beta}_{2} \cdot\left(\nabla \times \boldsymbol{\psi}_{2}\right) \quad \text { on } S_{12} \\
\boldsymbol{\psi}_{1} & =\boldsymbol{\psi}_{2} \quad \text { on } S_{12} \\
\alpha_{1} \nabla \cdot \boldsymbol{\psi}_{1} & =\alpha_{2} \nabla \cdot \boldsymbol{\psi}_{2} \quad \text { on } S_{12}
\end{aligned}
$$

Note the correspondence between the number of unknowns and the number of equations in $V$ and the boundary conditions on $S$.

\subsection{Variational formulations}

A weak form of the boundary value problem (18) can be derived by setting up the scalar product of an arbitrary test-function vector $\delta \psi$ with the field equation (18) 1 and integrating over $V$. The test-function has to satisfy the homogeneous versions of the Dirichlet boundary and interface conditions (18) $2,5,7$. Partial integration yields with account of the Neumann boundary conditions $(18)_{3,4,6,8}$ and the field equation $(18)_{1}$ :

$$
\int_{V}[(\nabla \times \delta \boldsymbol{\psi}) \cdot \boldsymbol{\beta} \cdot(\nabla \times \boldsymbol{\psi})+\alpha(\nabla \cdot \delta \boldsymbol{\psi})(\nabla \cdot \boldsymbol{\psi})] \mathrm{d} V-\int_{S_{E}} \delta \boldsymbol{\psi} \cdot \mathbf{E}_{S} \mathrm{~d} S=0
$$

The weak form, Equation (19), can be modified in order to incorporate directly a prescribed electric surface potential, $\phi=\phi_{s}$ on $S_{E}$ : Substituting $\mathbf{E}_{S}=-\mathbf{n} \times \nabla \phi$ into the surface integral over $S_{E}$, noting that $\delta \psi \cdot(\mathbf{n} \times \nabla \phi)$ vanishes identically on $S_{D}$ due to the Dirichlet boundary condition $(18)_{2}$ and extending the surface integration to $S_{D}$, we obtain after partial integration:

$$
\int_{V}[(\nabla \times \delta \boldsymbol{\psi}) \cdot \boldsymbol{\beta} \cdot(\nabla \times \boldsymbol{\psi})+\alpha(\nabla \cdot \delta \boldsymbol{\psi})(\nabla \cdot \boldsymbol{\psi})] \mathrm{d} V-\int_{S_{E}} \phi_{S} \mathbf{n} \cdot(\nabla \times \delta \boldsymbol{\psi}) \mathrm{d} S=0
$$

In the linear case, the solution of (20) can be obtained also by minimizing the functional

$$
F(\boldsymbol{\psi})=\frac{1}{2} \int_{V}\left[(\nabla \times \boldsymbol{\psi}) \cdot \boldsymbol{\beta} \cdot(\nabla \times \boldsymbol{\psi})+\alpha(\nabla \cdot \boldsymbol{\psi})^{2}\right] \mathrm{d} V-\int_{S_{E}} \phi_{S} \mathbf{n} \cdot(\nabla \times \boldsymbol{\psi}) \mathrm{d} S
$$

\subsection{FE formulations}

The vector potential written in vector-matrix notation as $\{\boldsymbol{\psi}(\mathbf{r})\}=\left\{\psi_{x}(\mathbf{r}), \psi_{y}(\mathbf{r}), \psi_{z}(\mathbf{r})\right\}^{\mathrm{T}}$ is interpolated from nodal quantities $\left\{\boldsymbol{\psi}^{n}\right\}$ using the shape functions $[\mathbf{N}(\mathbf{r})]$ as follows:

$$
\{\boldsymbol{\psi}\}=[\mathbf{N}]\left\{\boldsymbol{\psi}^{n}\right\}
$$

From now on, $\{$.$\} denote columns of the FE quantities, \{.\}^{\mathrm{T}}$ their transposition, whereas [.] are matrix operators acting on the columns. 
The electric displacement components $\{\mathbf{D}(\mathbf{r})\}$ can be derived from the nodal variables as

$$
\{\mathbf{D}\}=\left[\mathbf{B}^{\psi}\right]\left\{\boldsymbol{\psi}^{n}\right\}
$$

where the 'curl' matrix $\left[\mathbf{B}^{\psi}(\mathbf{r})\right]$ is defined as [3]

$$
\left[\mathbf{B}^{\psi}\right]=\left[\begin{array}{ccc}
0 & \partial_{z} & -\partial_{y} \\
-\partial_{z} & 0 & \partial_{x} \\
\partial_{y} & -\partial_{x} & 0
\end{array}\right][\mathbf{N}]
$$

The divergence of the vector potential is approximated by the relation

$$
\nabla \cdot \boldsymbol{\psi}=\{\mathbf{A}\}^{\mathrm{T}}\left\{\boldsymbol{\psi}^{n}\right\}
$$

where the 'divergence' vector $\{\mathbf{A}(\mathbf{r})\}$ is defined as

$$
\{\mathbf{A}\}^{\mathrm{T}}=\left\{\partial_{x} \partial_{y} \partial_{z}\right\}[\mathbf{N}]
$$

Equation (20) yields the FE equations

$$
\left(\left[\mathbf{K}^{\psi \psi}\right]+\left[\mathbf{K}^{\alpha}\right]\right)\left\{\boldsymbol{\psi}^{n}\right\}=\left\{\mathbf{Q}_{S}\right\}
$$

with the stiffness matrices $\left[\mathbf{K}^{\psi \psi}\right]$ and $\left[\mathbf{K}^{\alpha}\right]$ and the load vector $\left\{\mathbf{Q}_{S}\right\}$

$$
\begin{aligned}
{\left[\mathbf{K}^{\psi \psi}\right] } & =\int_{V}\left[\mathbf{B}^{\psi}\right]^{\mathrm{T}}[\boldsymbol{\beta}]\left[\mathbf{B}^{\psi}\right] \mathrm{d} V \\
{\left[\mathbf{K}^{\alpha}\right] } & =\int_{V} \alpha\{\mathbf{A}\}\{\mathbf{A}\}^{\mathrm{T}} \mathrm{d} V \\
\left\{\mathbf{Q}_{S}\right\} & =-\int_{S_{E}} \phi_{\mathrm{S}}\left[\mathbf{B}^{\psi}\right]^{\mathrm{T}}\{\mathbf{n}\} \mathrm{d} S
\end{aligned}
$$

\subsection{Electrical 'rigid body' degrees of freedoms}

Consider again the field equations including the Coulomb gauge condition of the boundary value problem (18). In combination with the boundary conditions, they yield a unique solution of the vector potential $\psi(\mathbf{r})$. In a particular FE model, the corresponding number of discrete Dirichlet boundary conditions $(18)_{2,5}$ may be reduced, however. In order to approach this question, we determined for several structures and gauge methods the number of zero-energy modes of the global stiffness matrix, which is equal to the minimum number of discrete nodal constraints or Dirichlet boundary conditions required for uniqueness. Table I shows the rank deficiency (number of zero eigenvalues) of the stiffness matrix for an ungauged formulation, Coulomb gauge and two-component gauge.

For the Coulomb gauge condition applied in $V$, the number of zero eigenvalues of the stiffness matrix remains equal to 9 for all considered structures, similar to the constant number of 6 rigid body motions in mechanics. The analogy is incomplete, however, as the number of 'electrical rigid body motions' depends on the FE type: For 20-node hexahedral elements not shown in Table I, the rank deficiency of the stiffness matrix was equal to 15 . For eightnode hexahedral elements with nine electrical rigid body motions, the zero-energy modes are 


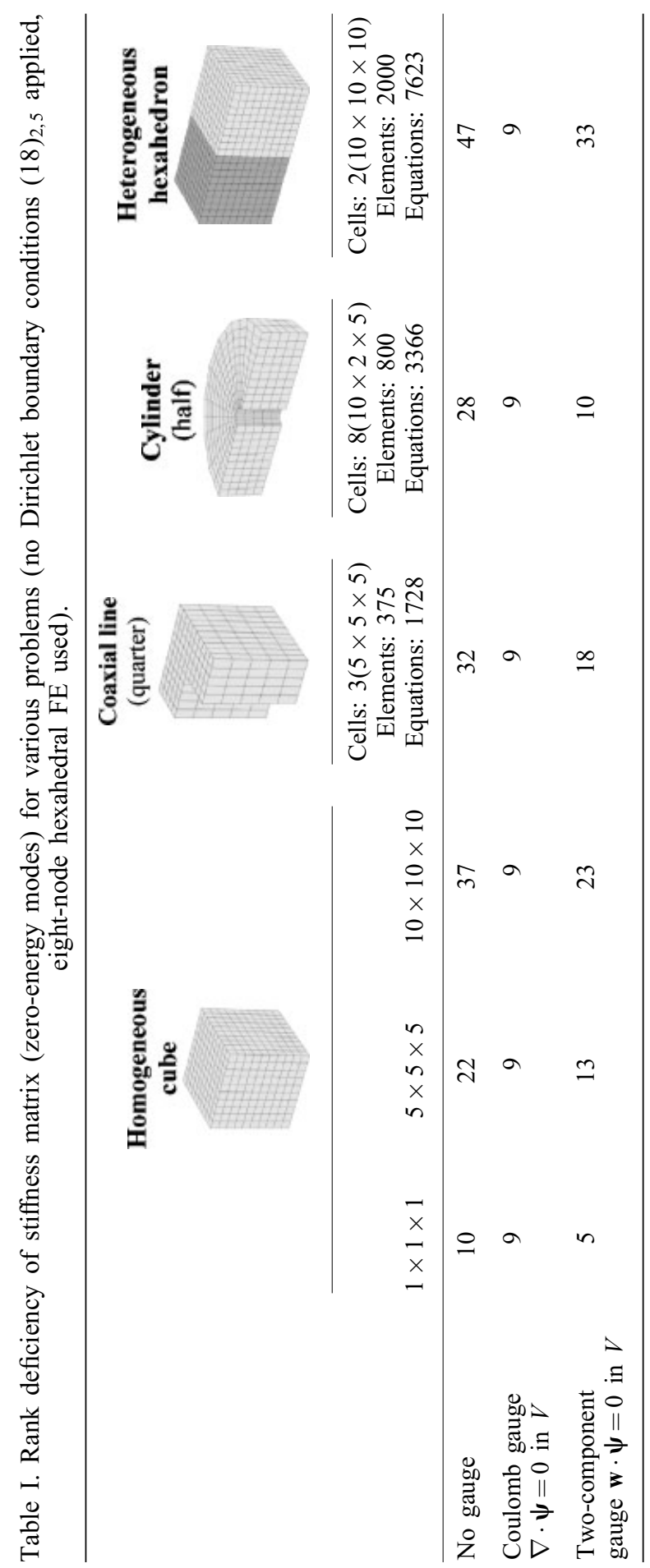




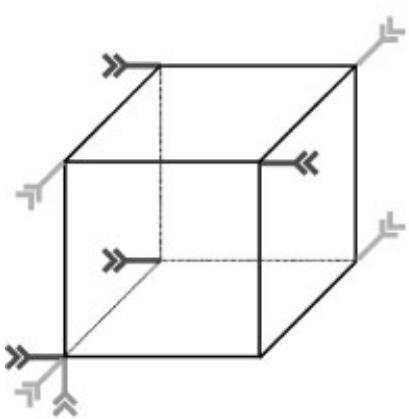

(a)

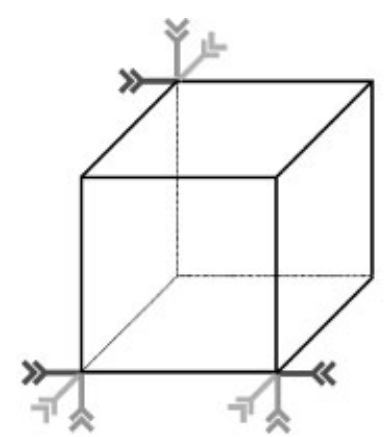

(b)

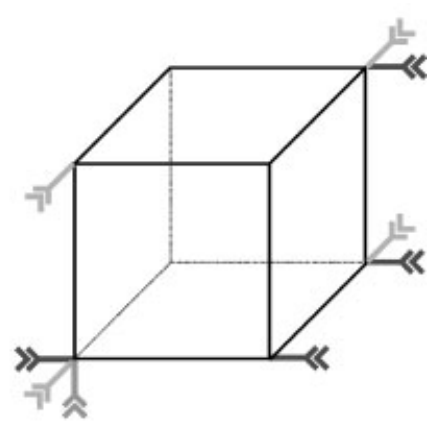

(c)

Figure 1. Electrical 'rigid body constraints' of the vector potential for an eight-node hexahedral element preventing rank deficiency of the stiffness matrix.

three 'translations', four 'shear modes' and two modes of biaxial 'elongation-shrinkage' under constant 'volume'. Some examples of electrical constraints removing the rank deficiency of the stiffness matrix are given in Figure 1 for a single eight-node element.

\section{THE VECTOR POTENTIAL IN PIEZOELECTRICITY}

\subsection{Coupled electromechanical boundary value problem}

The field equations of electromechanical equilibrium and compatibility in the absence of volume charges are

$$
\begin{aligned}
\nabla \cdot \boldsymbol{\sigma}+\mathbf{f}_{V} & =\mathbf{0} \\
\boldsymbol{\varepsilon} & =(\nabla \mathbf{u})^{S} \quad \text { in } V \\
\nabla \cdot \mathbf{D} & =0 \\
\nabla \times \mathbf{E} & =\mathbf{0}
\end{aligned}
$$

where $\boldsymbol{\sigma}, \boldsymbol{\varepsilon}$ and $\mathbf{u}$ denote stress, strain and displacements, the strain given by the symmetric part of the displacement gradient in the small strain limit; $\mathbf{f}_{v}$ is the body force vector. Possible associated boundary conditions are

$$
\begin{aligned}
& \mathbf{n} \cdot \boldsymbol{\sigma}=\mathbf{f}_{S} \quad \text { on } S_{\sigma} \\
& \mathbf{u}=\mathbf{u}_{S} \quad \text { on } S_{u} \\
& \mathbf{n} \cdot \mathbf{D}=D_{n} \quad \text { on } S_{D} \\
& \mathbf{n} \times \mathbf{E}=\mathbf{E}_{S} \quad \text { on } S_{E}
\end{aligned}
$$

where the surface $S$ is composed of $S=S_{\sigma} \cup S_{u}\left(S_{\sigma} \cap S_{u}=\emptyset\right)$, and $\mathbf{f}_{S}$ and $\mathbf{u}_{S}$ are the prescribed surface tractions and displacements. Equations (29) and (30) have to be complemented by 
constitutive relations. In piezoelectric materials, these can be specified as

$$
\begin{aligned}
& \boldsymbol{\sigma}={ }^{4} \mathbf{C}^{D} \cdot \cdot\left(\boldsymbol{\varepsilon}-\boldsymbol{\varepsilon}^{r}\right)-\left(\mathbf{D}-\mathbf{P}^{r}\right) \cdot{ }^{3} \mathbf{h} \\
& \mathbf{E}=-{ }^{3} \mathbf{h} \cdot \cdot\left(\boldsymbol{\varepsilon}-\boldsymbol{\varepsilon}^{r}\right)+\boldsymbol{\beta}^{\varepsilon} \cdot\left(\mathbf{D}-\mathbf{P}^{r}\right)
\end{aligned}
$$

or

$$
\begin{aligned}
\boldsymbol{\sigma} & ={ }^{4} \mathbf{C}^{E} \cdot \cdot\left(\boldsymbol{\varepsilon}-\boldsymbol{\varepsilon}^{r}\right)-\mathbf{E} \cdot{ }^{3} \mathbf{e} \\
\mathbf{D}-\mathbf{P}^{r} & ={ }^{3} \mathbf{e} \cdot \cdot\left(\boldsymbol{\varepsilon}-\boldsymbol{\varepsilon}^{r}\right)+\boldsymbol{\kappa}^{\varepsilon} \cdot \mathbf{E}
\end{aligned}
$$

where the fourth rank tensors ${ }^{4} \mathbf{C}^{D}$ and ${ }^{4} \mathbf{C}^{E}$, the third rank tensors ${ }^{3} \mathbf{h}$ and ${ }^{3} \mathbf{e}$, and the second rank tensors $\boldsymbol{\beta}^{\varepsilon}$ and $\boldsymbol{\kappa}^{\varepsilon}$ are material tensors characterizing mechanical stiffness, piezoelectricity and dielectric moduli, respectively; $\boldsymbol{\varepsilon}^{r}$ and $\mathbf{P}^{r}$ are the remanent strain and the remanent polarization. The material constants and the two sets of constitutive relations in Equations (31) and (32) are equivalent and can be converted into each other by linear transformations. In the linear load range (roughly speaking below the coercive electric field and the coercive mechanical stress), the material tensors and remanent properties are load independent functions of position. In the nonlinear load range, they depend in a nonlinear fashion on the load history. Formulations of the boundary value problem in terms of the electric vector potential, Equation (4), or in terms of the scalar potential, Equation (3), call for the constitutive relations (31) or (32), respectively.

Extending the results of the preceding section, we can formulate the electromechanical boundary value problem for the displacements and the electric vector potential with penalized Coulomb gauge:

$$
\begin{aligned}
\nabla \cdot \boldsymbol{\sigma}+\mathbf{f}_{V} & =\mathbf{0} \quad \text { in } V \\
\nabla \times \mathbf{E}+\nabla(\alpha \nabla \cdot \boldsymbol{\psi}) & =\mathbf{0} \quad \text { in } V \\
\mathbf{n} \cdot \boldsymbol{\sigma} & =\mathbf{f}_{S} \quad \text { on } S_{\sigma} \\
\mathbf{u} & =\mathbf{u}_{S} \quad \text { on } S_{u} \\
\mathbf{n} \times \boldsymbol{\psi} & =\mathbf{P} \quad \text { on } S_{D} \\
\alpha \nabla \cdot \boldsymbol{\psi} & =0 \quad \text { on } S_{D} \\
\mathbf{n} \times \mathbf{E} & =\mathbf{E}_{S} \quad \text { on } S_{E} \\
\mathbf{n} \cdot \boldsymbol{\psi} & =0 \quad \text { on } S_{E} \\
\mathbf{n} \cdot \boldsymbol{\sigma}_{1} & =\mathbf{n} \cdot \boldsymbol{\sigma}_{2} \quad \text { on } S_{12} \\
\mathbf{u}_{1} & =\mathbf{u}_{2} \quad \text { on } S_{12} \\
\mathbf{n} \times \mathbf{E}_{1} & =\mathbf{n} \times \mathbf{E}_{2} \quad \text { on } S_{12} \\
\boldsymbol{\psi}_{1} & =\boldsymbol{\psi}_{2} \quad \text { on } S_{12} \\
\alpha_{1} \nabla \cdot \boldsymbol{\psi}_{1} & =\alpha_{2} \nabla \cdot \boldsymbol{\psi}_{2} \quad \text { on } S_{12}
\end{aligned}
$$


where stress and electric field have to be expressed by

$$
\begin{aligned}
& \boldsymbol{\sigma}={ }^{4} \mathbf{C}^{D} \cdot \cdot\left[(\nabla \mathbf{u})^{S}-\boldsymbol{\varepsilon}^{r}\right]+\left[(\nabla \times \boldsymbol{\psi})+\mathbf{P}^{r}\right] \cdot{ }^{3} \mathbf{h} \\
& \mathbf{E}=-{ }^{3} \mathbf{h} \cdot \cdot\left[(\nabla \mathbf{u})^{S}-\boldsymbol{\varepsilon}^{r}\right]-\boldsymbol{\beta}^{\varepsilon} \cdot\left[(\nabla \times \boldsymbol{\psi})+\mathbf{P}^{r}\right]
\end{aligned}
$$

\subsection{Variational formulations}

The FE equations of coupled electromechanical boundary value problems can be derived from electromechanical variational principles generalizing the principle of virtual work in mechanics. In the standard approach, the electric field is expressed by the scalar potential according to Equation (3) [2], and the variational formulation is written as

$$
\int_{V}(\boldsymbol{\sigma} \cdot \delta \boldsymbol{\varepsilon}-\mathbf{D} \cdot \delta \mathbf{E}) \mathrm{d} V=\int_{V} \mathbf{f}_{V} \cdot \delta \mathbf{u} \mathrm{d} V+\int_{S}(\mathbf{n} \cdot \boldsymbol{\sigma} \cdot \delta \mathbf{u}+\mathbf{n} \cdot \mathbf{D} \delta \phi) \mathrm{d} S
$$

In this case, the displacements $\mathbf{u}$ and the scalar potential $\phi$ are the basic variables, $\delta \boldsymbol{\varepsilon}=(\nabla \delta \mathbf{u})^{S}$ and $\delta \mathbf{E}=-\nabla \delta \phi$.

In the alternate approach, the electric induction is expressed by the vector potential according to (4) [3]. The principle of virtual work can be stated in the form

$$
\int_{V}(\boldsymbol{\sigma} \cdot \delta \boldsymbol{\varepsilon}+\mathbf{E} \cdot \delta \mathbf{D}) \mathrm{d} V=\int_{V} \mathbf{f}_{V} \cdot \delta \mathbf{u} \mathrm{d} V+\int_{S}(\mathbf{n} \cdot \boldsymbol{\sigma} \cdot \delta \mathbf{u}+\mathbf{n} \times \mathbf{E} \cdot \delta \boldsymbol{\psi}) \mathrm{d} S
$$

where $\delta \mathbf{D}=-\nabla \times \delta \psi$, as now the components of the vector potential are the basic electric variables.

Satisfaction of Equation (35) for arbitrary variations $\delta \mathbf{u}$ and $\delta \phi$ implies the mechanical and electrical equilibrium (29) $)_{1,3}$, as shown by application of Gauss' theorem. Similarly, the system is in the mechanical and electrical equilibrium (29) $)_{1,4}$ if Equation (36) holds for arbitrary variations $\delta \mathbf{u}$ and $\delta \psi$.

The volume integral on the left-hand side in Equation (35) represents the variation of a mixed potential - energy in the mechanical sense, but enthalpy in the electrical sense. Therefore, solutions of boundary value problems correspond to saddle points of the potential as a function of $\mathbf{u}$ and $\phi$. The volume integral on the left-hand side in Equation (36), on the other hand, is a pure electromechanical energy variation, rendering solutions of the boundary value problem to true minima of the potential as a function of $\mathbf{u}$ and $\psi$. In the context of the FE method, a consequence of the minimum property of the energy potential are positive definite stiffness matrices, which are of advantage in the case of nonlinear problems. For linear problems both formulations are equivalent up to numerical errors, except that the number of the nodal degrees of freedom is larger in case of the vector potential.

In order to achieve uniqueness of the vector potential in coupled electromechanical problems, we suggest to extend the penalized Coulomb gauge condition of the preceding section to this case. The modified variational principle (36) is given by

$$
\begin{aligned}
& \int_{V} {[\boldsymbol{\sigma} \cdot \delta \boldsymbol{\varepsilon}+\mathbf{E} \cdot \delta \mathbf{D}+\alpha(\nabla \cdot \boldsymbol{\psi})(\nabla \cdot \delta \boldsymbol{\psi})] \mathrm{d} V } \\
& \quad=\int_{V} \mathbf{f}_{V} \cdot \delta \mathbf{u} \mathrm{d} V+\int_{S}[\mathbf{n} \cdot \boldsymbol{\sigma} \cdot \delta \mathbf{u}+\mathbf{n} \times \mathbf{E} \cdot \delta \boldsymbol{\psi}+\alpha(\nabla \cdot \boldsymbol{\psi}) \mathbf{n} \cdot \delta \boldsymbol{\psi}] \mathrm{d} S
\end{aligned}
$$


With account of the boundary conditions in (33), the variational principle takes the form

$$
\int_{V}[\boldsymbol{\sigma} \cdot \delta \boldsymbol{\varepsilon}+\mathbf{E} \cdot \delta \mathbf{D}+\alpha(\nabla \cdot \boldsymbol{\psi})(\nabla \cdot \delta \boldsymbol{\psi})] \mathrm{d} V=\int_{V} \mathbf{f}_{V} \cdot \delta \mathbf{u} \mathrm{d} V+\int_{S_{\sigma}} \mathbf{f}_{S} \cdot \delta \mathbf{u} \mathrm{d} S+\int_{S_{E}} \mathbf{E}_{S} \cdot \delta \boldsymbol{\psi} \mathrm{d} S
$$

Similar to the transition from Equations (19) to (20) the electrical work on the surface $S_{E}$ can be expressed directly by a prescribed electric surface potential $\phi_{s}$

$$
\begin{aligned}
& \int_{V} {[\boldsymbol{\sigma} \cdot \delta \boldsymbol{\varepsilon}+\mathbf{E} \cdot \delta \mathbf{D}+\alpha(\nabla \cdot \boldsymbol{\psi})(\nabla \cdot \delta \boldsymbol{\psi})] \mathrm{d} V } \\
&=\int_{V} \mathbf{f}_{V} \cdot \delta \mathbf{u} \mathrm{d} V+\int_{S_{\sigma}} \mathbf{f}_{S} \cdot \delta \mathbf{u} \mathrm{d} S+\int_{S_{E}} \phi_{S} \mathbf{n} \cdot(\nabla \times \delta \boldsymbol{\psi}) \mathrm{d} S
\end{aligned}
$$

The variational formulation (39) provides equations in $V$ and a set of natural boundary conditions corresponding to the boundary value problem (33).

\subsection{FE formulation}

To generate the piezoelectric matrix relations for a FE, the continuous displacement vector $\{\mathbf{u}(\mathbf{r})\}=\left\{u_{x}(\mathbf{r}), u_{y}(\mathbf{r}), u_{z}(\mathbf{r})\right\}^{\mathrm{T}}$ and vector potential $\{\boldsymbol{\psi}(\mathbf{r})\}=\left\{\psi_{x}(\mathbf{r}), \psi_{y}(\mathbf{r}), \psi_{z}(\mathbf{r})\right\}^{\mathrm{T}}$ are expressed by the nodal values $\left\{\mathbf{u}^{n}\right\}$ and $\left\{\boldsymbol{\psi}^{n}\right\}$ via shape functions $\left[\mathbf{N}^{u}(\mathbf{r})\right]$ and $\left[\mathbf{N}^{\psi}(\mathbf{r})\right]$, respectively

$$
\begin{aligned}
\{\mathbf{u}\} & =\left[\mathbf{N}^{u}\right]\left\{\mathbf{u}^{n}\right\} \\
\{\boldsymbol{\psi}\} & =\left[\mathbf{N}^{\psi}\right]\left\{\boldsymbol{\psi}^{n}\right\}
\end{aligned}
$$

In a similar manner, the prescribed body and surface forces and charges are interpolated from nodal quantities using the shape functions.

Differentiation of Equation (40) yields the following expression for the strains $\{\boldsymbol{\varepsilon}(\mathbf{r})\}=\left\{\varepsilon_{x}(\mathbf{r})\right.$, $\left.\varepsilon_{y}(\mathbf{r}), \varepsilon_{z}(\mathbf{r}), \gamma_{x y}(\mathbf{r}), \gamma_{y z}(\mathbf{r}), \gamma_{z x}(\mathbf{r})\right\}^{\mathrm{T}}$

$$
\{\boldsymbol{\varepsilon}\}=\left[\mathbf{B}^{u}\right]\left\{\mathbf{u}^{n}\right\}
$$

where $\left[\mathbf{B}^{u}(\mathbf{r})\right]$ is the 'gradient' matrix [4]. The corresponding equation for the electric displacement $\{\mathbf{D}\}$ is given by (23).

The FE equations follow by substitution of the constitutive relations (34) and of Equations (40)-(42), (23), (25) into the variational principle (39)

$$
\begin{aligned}
{\left[\mathbf{K}^{u u}\right]\left\{\mathbf{u}^{n}\right\}+\left[\mathbf{K}^{u \psi}\right]\left\{\boldsymbol{\psi}^{n}\right\} } & =\left\{\mathbf{F}_{r}\right\}+\left\{\mathbf{F}_{V}\right\}+\left\{\mathbf{F}_{S}\right\} \\
{\left[\mathbf{K}^{\psi u}\right]\left\{\mathbf{u}^{n}\right\}+\left(\left[\mathbf{K}^{\psi \psi}\right]+\left[\mathbf{K}^{\alpha}\right]\right)\left\{\boldsymbol{\psi}^{n}\right\} } & =\left\{\mathbf{Q}_{r}\right\}+\left\{\mathbf{Q}_{S}\right\}
\end{aligned}
$$

where the stiffness matrices are given by

$$
\begin{array}{ll}
{\left[\mathbf{K}^{u u}\right]=\int_{V}\left[\mathbf{B}^{u}\right]^{\mathrm{T}}\left[\mathbf{C}^{D}\right]\left[\mathbf{B}^{u}\right] \mathrm{d} V,} & {\left[\mathbf{K}^{u \psi}\right]=-\int_{V}\left[\mathbf{B}^{u}\right]^{\mathrm{T}}[\mathbf{h}]^{\mathrm{T}}\left[\mathbf{B}^{\psi}\right] \mathrm{d} V} \\
{\left[\mathbf{K}^{\psi u}\right]=-\int_{V}\left[\mathbf{B}^{\psi}\right]^{\mathrm{T}}[\mathbf{h}]\left[\mathbf{B}^{u}\right] \mathrm{d} V,} & {\left[\mathbf{K}^{\psi \psi}\right]=\int_{V}\left[\mathbf{B}^{\psi}\right]^{\mathrm{T}}\left[\boldsymbol{\beta}^{\varepsilon}\right]\left[\mathbf{B}^{\psi}\right] \mathrm{d} V, \quad\left[\mathbf{K}^{\alpha}\right]=\int_{V} \alpha\{\mathbf{A}\}\{\mathbf{A}\}^{\mathrm{T}} \mathrm{d} V}
\end{array}
$$


and the load vectors are

$$
\begin{aligned}
\left\{\mathbf{F}_{r}\right\} & =\int_{V}\left[\mathbf{B}^{u}\right]^{\mathrm{T}}\left\{\boldsymbol{\sigma}^{\mathrm{r}}\right\} \mathrm{d} V, \quad\left\{\mathbf{F}_{V}\right\}=\int_{V}\left[\mathbf{N}^{u}\right]^{\mathrm{T}}\left\{\mathbf{f}_{\mathrm{V}}\right\} \mathrm{d} V, \quad\left\{\mathbf{F}_{S}\right\}=\int_{S_{\sigma}}\left[\mathbf{N}^{u}\right]^{\mathrm{T}}\left\{\mathbf{f}_{\mathrm{S}}\right\} \mathrm{d} S \\
\left\{\mathbf{Q}_{r}\right\} & =\int_{V}\left[\mathbf{B}^{\psi}\right]^{\mathrm{T}}\left\{\mathbf{E}^{\mathrm{r}}\right\} \mathrm{d} V, \quad\left\{\mathbf{Q}_{S}\right\}=-\int_{S_{E}}\left[\mathbf{B}^{\psi}\right]^{\mathrm{T}} \phi_{\mathrm{S}}\{\mathbf{n}\} \mathrm{d} S
\end{aligned}
$$

where

$$
\begin{aligned}
\left\{\boldsymbol{\sigma}^{r}\right\} & =\left[\mathbf{C}^{D}\right]\left\{\boldsymbol{\varepsilon}^{r}\right\}-[\mathbf{h}]^{\mathrm{T}}\left\{\mathbf{P}^{r}\right\} \\
\left\{\mathbf{E}^{r}\right\} & =-[\mathbf{h}]\left\{\boldsymbol{\varepsilon}^{r}\right\}+\left[\boldsymbol{\beta}^{\varepsilon}\right]\left\{\mathbf{P}^{r}\right\}
\end{aligned}
$$

In order to avoid confusion, note our definition of the matrix of piezoelectricity $\left[h_{k \alpha}\right]$ with $k=1 \ldots 3$ and $\alpha=1 \ldots 6$, which retains the sequence of indices of the piezoelectric tensor $h_{k i j}$-first the 'electric' index $k$ followed by the 'mechanical' indices $i$ and $j$ or $\alpha$. Allik and Hughes [2] and Landis [3] used the opposite convention, adopting the transition rule $h_{k i j} \rightarrow\left[h_{\alpha k}\right]$.

Equations (43)-(46) represent an extension of Landis' formulation [3] modified by addition of the penalty term in order to obtain an unique solution of the vector potential in threedimensional problems. The case $\alpha=0$ corresponds to the ungauged formulation with a singular stiffness matrix, which requires reduced integration [4] with low-order numerical integration rules. This leads, however, to slow convergence (high number of conjugate gradient iterations) for iterative methods of the FE system solution or to a possibly decreasing accuracy of the solution obtained by direct methods.

\section{NUMERICAL EXAMPLES}

The electric scalar and vector potential formulations described above have been implemented into the FE program PANTOCRATOR [18] using 8-node and 20-node hexahedral FE. The three-dimensional problems reported below compare gauged and ungauged vector potential formulations for linear-dielectric materials and a nonlinear ferroelectric material. The first two examples involving linear-dielectric materials investigate the computational difficulties in structures with abrupt changes of the material properties and with a reentrant corner, which have been noted by Demerdash and Wang [19] and Kaltenbacher and Reitzinger [20] in the context of the magnetic vector potential. The third example concerns the nonlinear analysis of a ferroelectric polycrystalline ceramics.

\subsection{Dielectric inhomogeneity subject to a homogeneous far field}

Consider a small cube with edge length $2 a$ and an isotropic dielectric constant $\kappa_{1}$ embedded in a medium with a dielectric constant $\kappa_{2}$ subject to an electric far field induction $D_{0}$ in $z$ direction. In the FE model, the infinite medium is represented by a cube of dimensions $2 b \gg 2 a$ (Figure 2). A similar geometry has been used in References [5,9] for eddy current and magnetostatic analysis. Due to symmetry, only one-eighth of the problem is presented. The planes $x=0, y=0, z=0$ are symmetry planes, whereas the planes $x=b, y=b, z=b$ 


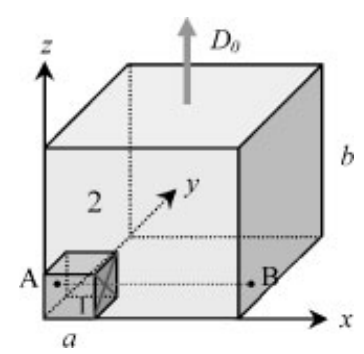

Figure 2. Dielectric inhomogeneity subjected to a homogeneous electric far field induction $D_{0}$.

Table II. Boundary conditions on the electric vector potential used in example 1 .

\begin{tabular}{ll}
\hline$x=0$ & $\psi_{y}=0, \psi_{z}=0$ \\
$x=b$ & $\psi_{y}=-D_{0} b / 2, \psi_{z}=0$ \\
$y=0$ & $\psi_{x}=0, \psi_{z}=0$ \\
$y=b$ & $\psi_{x}=D_{0} b / 2, \psi_{z}=0$ \\
$z=0$ & $\psi_{z}=0$ \\
$z=b$ & $\psi_{z}=0$ \\
\hline
\end{tabular}

represent far boundaries. The far field $D_{0}$ has been simulated by the Dirichlet boundary conditions for the vector potential summarized in Table II.

The computations have been carried out using eight-node isoparametric brick elements. The problem region has been subdivided into $8000 \mathrm{FE}$ containing 9261 nodes (Figure 5). The dielectric permittivity of the inhomogeneity is much larger than that of the surrounding medium, $\kappa_{1}=1000 \kappa_{2}$. A uniform penalty value $\alpha=1 / \kappa_{1}$ is used for both materials. The comparison of the solutions based on the scalar potential, gauged and ungauged vector potential demonstrates a decrease of the accuracy with growing penalty value, see Figure 3 for the distribution of the three components of $\mathbf{D}$ along the line $y=z=a / 2$ (line $\mathrm{AB}$ in Figure 2). The most significant differences are observed for the $D_{y}$ component.

Various choices of the penalty value are possible for problems with material inhomogeneities. In the magnetostatic analysis of Reference [7], different penalty values were used for each material corresponding to $\alpha_{i}=1 / \kappa_{i}$. However, our results with various penalty strategies for a wide range of dielectric permittivity ratios indicate that a constant single penalty value for all materials $\alpha=1 / \kappa_{\max }$ is to be preferred (Figure 4 ) which can be rationalized as follows: In the case of $\alpha_{i}=1 / \kappa_{i}$, the discontinuity in $\alpha$ implies according to (18) 8 a discontinuity of $\nabla \cdot \psi$ which is never strictly zero due to the limited numerical precision of the FE solution. In other words, a small value of $\nabla \cdot \psi$ in medium 2 with a low $\kappa$ leads to a large value of $\nabla \cdot \psi$ in medium 1 with a high $\kappa$, and thus, taking into account $(18)_{1}$, it is impossible to achieve a sufficient accuracy for $\mathbf{D}$.

Inaccurate results induced by gauging have been observed mainly in problems with considerable jumps of the dielectric permittivity, whereas the accuracy of the solution in homogeneous materials was not influenced. The error due to gauging can be reduced by mesh refinement 

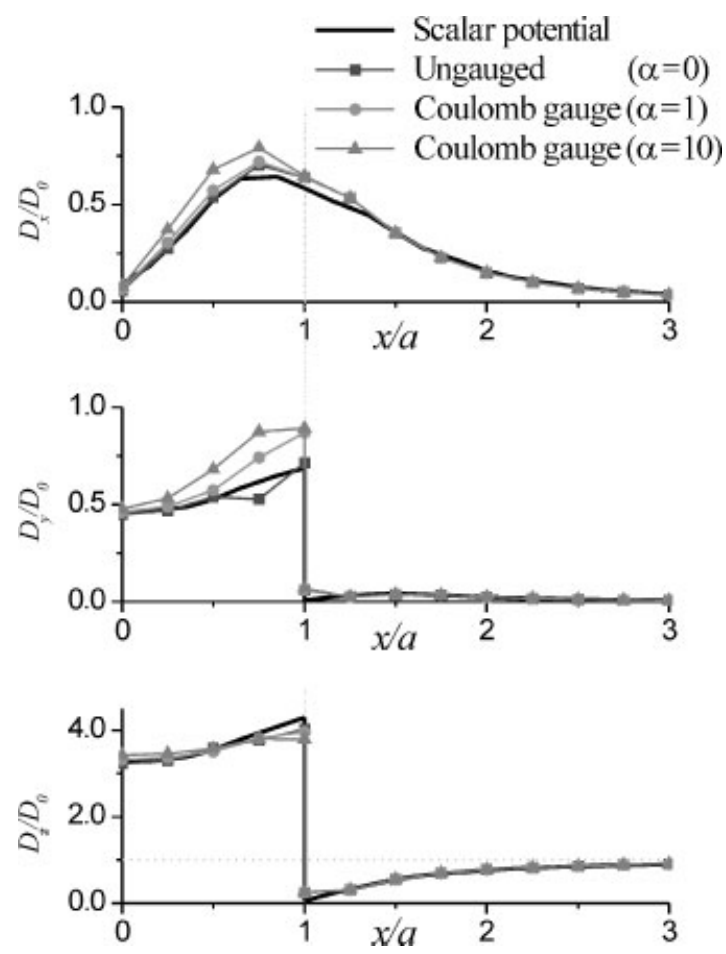

Figure 3. Electric displacement components along the line $y=z=a / 2$ intersecting the interface.

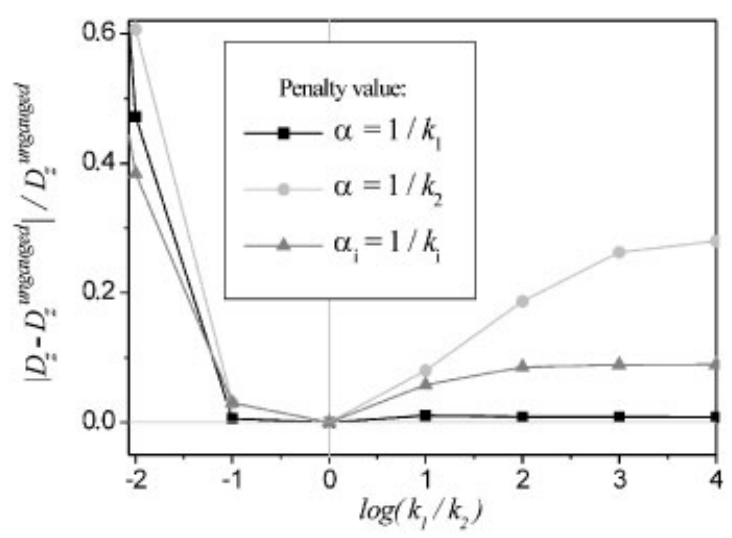

Figure 4. Comparison of various strategies for the choice of penalty value.

due to $\nabla \cdot \psi$ decreasing for smaller element sizes (compare top and bottom rows of pictures in Figure 5). A nonvanishing field of $\nabla \cdot \psi$ localizes along the interface between the two media (Figure 5), and with a finer mesh it decays faster into the volume. The maximum value of $\nabla \cdot \psi$ also decreases with a growing penalty factor $\alpha$. 


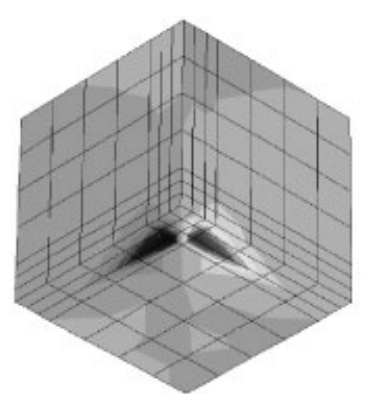

$\alpha=0, \quad 343$ elements

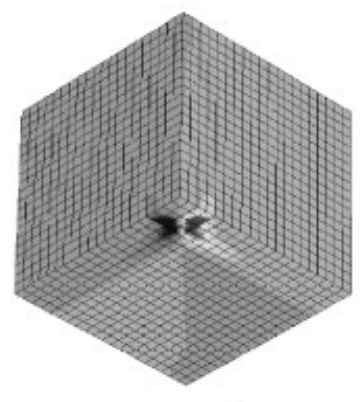

$\alpha=0, \quad 8000$ elements

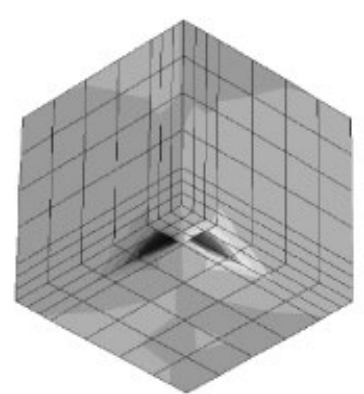

$\alpha=1, \quad 343$ elements

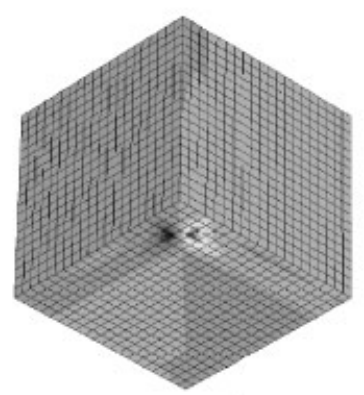

$\alpha=1, \quad 8000$ elements

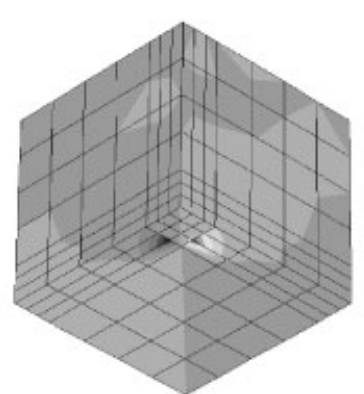

$\alpha=100, \quad 343$ elements

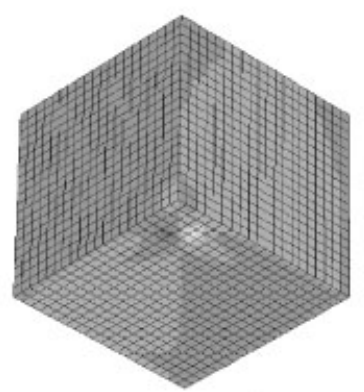

$\alpha=100, \quad 8000$ elements

Figure 5. Distribution of $\nabla \cdot \psi$ for different penalty values $\alpha$ and meshes.

\subsection{Cube partially embedded in a second material subject to an average field $D_{0}$}

In the second example, a cube of material 1 is surrounded from four sides by material 2 ('reentrant corner', Figure 6). The whole block is subjected to an average field $D_{0}$ in $z$ direction imposed by Dirichlet boundary conditions for the vector potential similar to the previous example (Table II). The planes $x=0, y=0, z=0$ are symmetry planes, and only oneeighth of the problem is presented. A similar geometry has been considered in Reference [20] in a magnetostatic analysis. The permittivity ratio is again $\kappa_{1} / \kappa_{2}=1000$, and a homogeneous penalty value $\alpha=1 / \kappa_{1}$ was used in both materials.

The analysis results for the gauged vector potential demonstrate a sensitivity to the type of the FE with remarkable differences between the field distributions around the reentrant corner obtained by linear 8-node or quadratic 20-node elements. The distribution of $D_{z}$ along the line $y=z=a$ (ABC in Figure 6) is shown in Figure 7. Computations with 20-node elements without gauge provide homogeneous fields in material 1 and 2 . The gauged formulation with 20-node elements leads to false oscillations near to the interface. The eight-node element formulation is free of oscillations, but demonstrates a regular drift from the exact solution both with and without gauging.

\subsection{Ferroelectric polycrystalline ceramics}

At large electric fields and/or stresses, piezoelectric materials are able to switch their polarization direction and thus demonstrate a highly nonlinear ferroelectric behaviour. Our motivation 


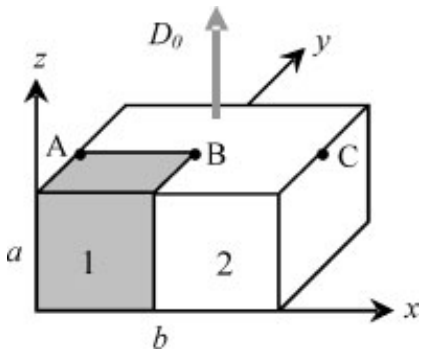

Figure 6. Heterogeneous dielectric block subjected to an average electric induction $D_{0}$.

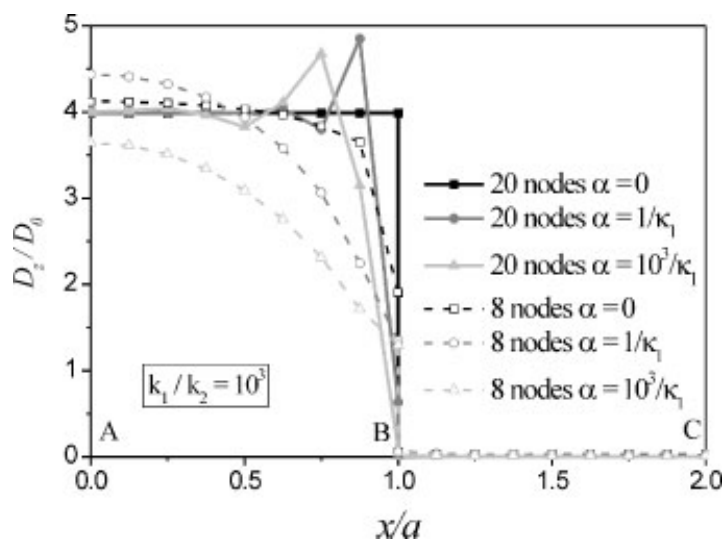

Figure 7. Distribution of electric displacement $D_{z}$ along the line ABC.

behind the analysis described below is to simulate the nonlinear constitutive behaviour of ferroelectric polycrystals by averaging the response of a sufficiently large number of single crystals in a representative volume element (RVE), which represents the macroscopic material point. The RVE consists of a cube with 8000 single crystals (Plate 1). The behaviour of each single crystal was modelled by a modified scheme adapted from Reference [21], which is based on direct modelling of domain wall motion in the single crystals.

Typical distributions of stress and electric field are given in Plate 1. The polycrystalline cube was subjected to an electric far field induction $D_{0}=0.045 \mathrm{C} / \mathrm{m}^{2}$ in $x$ direction. All outer sides were mechanically fixed. An incremental procedure employing modified NewtonRaphson iteration [4] was used in the nonlinear ferroelectric FE analysis. The advantage of gauging becomes apparent in the convergence of the iteration procedure: Figure 8 shows the relative error, which decreases monotonically to zero in the case of Coulomb gauging while the ungauged formulation does not converge due to nonuniqueness of the vector potential.

The results obtained point out the capability of the proposed approach to examine the coupled electromechanical behaviour of ferroelectric polycrystals in 3D. A quantitative analysis requires further comparison with experimental data. 


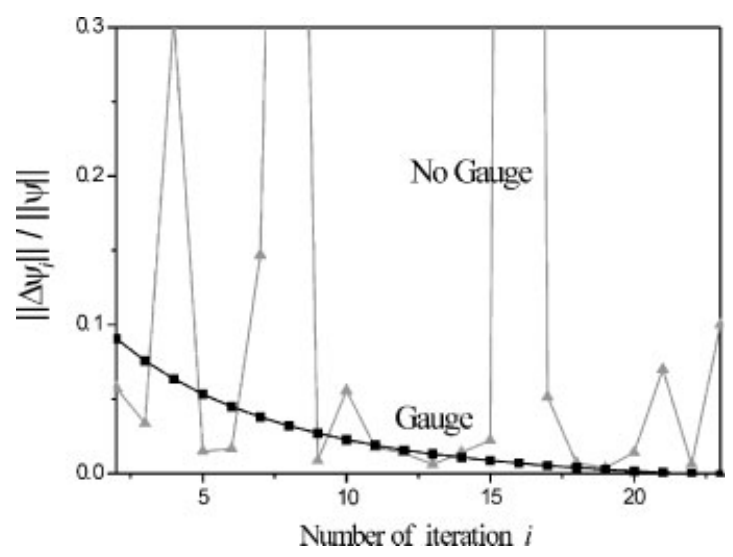

Figure 8. Convergence of iteration processes for gauged and ungauged formulations.

\section{CONCLUDING REMARKS}

FE formulations based on the electric vector potential represent an efficient tool to solve nonlinear coupled electromechanical problems. For three-dimensional problems, the vector potential requires some modifications caused by the loss of uniqueness. In the present paper, we have investigated the Coulomb gauge condition and appropriate boundary conditions that ensure an unique vector potential. Other variants of gauging procedures are briefly considered.

The Coulomb gauge condition has been satisfied by application of the penalty function method. Weak forms of three-dimensional electrostatic and piezoelectric boundary value problems with penalized Coulomb gauge are proposed and implemented in the FE program PANTOCRATOR.

For electrostatic problems, the minimum number of discrete Dirichlet boundary conditions, required to eliminate the electrical analogue of mechanical rigid body degrees of freedom, has been determined by spectral analysis of the stiffness matrix.

The influence of the penalty value on the accuracy of the FE solution has been analysed, for example problems with heterogeneous material properties and some practical recommendations are given.

\section{ACKNOWLEDGEMENT}

This work was supported by the German Research Foundation (DFG).

\section{REFERENCES}

1. Jackson JD. Classical Electrodynamics (3rd edn). Wiley: New York, 1998.

2. Allik H, Hughes TJR. Finite element method for piezoelectric vibration. International Journal for Numerical Methods in Engineering 1970; 2:151-157.

3. Landis CM. A new finite-element formulation for electromechanical boundary value problems. International Journal for Numerical Methods in Engineering 2002; 55:613-628.

4. Zienkiewicz OC, Taylor RL. The Finite Element Method, 1: The Basis (5th edn). Butterworth-Heinemann: Stoneham, MA, 2000. 
5. Biro O, Preis K. On the use of the magnetic vector potential in the finite-element analysis of three-dimensional eddy currents. IEEE Transactions on Magnetics 1989; 25:3145-3159.

6. Coulomb JL. Finite element three dimensional magnetic field computation. IEEE Transactions on Magnetics 1981; 17:3241-3246.

7. Jin J. The Finite Element Method in Electromagnetics. Wiley: New York, 1993.

8. Albertz D, Dappen S, Henneberger G. Calculation of 3D non-linear eddy current field in moving conductors and its application to braking systems. IEEE Transactions on Magnetics 1996; 32:768-771.

9. Mesquita RC, Bastos JPA. An incomplete gauge formulation for 3D nodal finite-element magnetostatics. IEEE Transactions on Magnetics 1992; 28:1044-1047.

10. Kangro U, Nicolaides R. Divergence boundary conditions for vector Helmholtz equations with divergence constraints. Mathematical Modelling and Numerical Analysis 1999; 33:479-492.

11. Carpenter CJ. Comparison of alternative formulations of 3-dimensional magnetic-field and eddy current problems at power frequencies. Proceedings of IEE 1977; 124:1024-1034.

12. Nishiguchi I, Matsuzawa H, Sasaki M. A comparative study of magnetostatics using three-field formulation and conventional vector potential formulations. IEEE Transactions on Magnetics 1997; 33:1243-1246.

13. Albanese R, Rubinacci G. Solution of three dimensional eddy current problems by integral and differential methods. IEEE Transactions on Magnetics 1988; 24:98-101.

14. Baumgart J. Constraining the finite element stiffness matrix in the vector potential formulation for electrostatics. Grosser Beleg, University of California, Santa Barbara, 2003.

15. Demerdash NA, Nehl TW, Fouad FA. Finite element formulation and analysis of three dimensional magnetic field problems. IEEE Transactions on Magnetics 1980; 16:1092-1094.

16. Helmholtz H. Über Integrale der hydrodynamischen Gleichungen, welche den Wirbelbewegungen entsprechen. Crelles Journal 1858; 55:25-55.

17. Spivak A. Comprehensive Introduction to Differential Geometry (3rd edn), vol. 3. Clothbound, 1999.

18. Semenov AS. PANTOCRATOR - the finite element program specialized on the nonlinear problem solution. Proceedings of the $V$ International Conference on 'Scientific and Engineering Problems of Predicting the Reliability and Service Life of Structures and Methods of their Solution', 2003; 466-480 (in Russian).

19. Demerdash NA, Wang R. Theoretical and numerical difficulties in $3 \mathrm{D}$ vector potential methods in finite element magnetostatic computations. IEEE Transactions on Magnetics 1990; 26:1656-1658.

20. Kaltenbacher M, Reitzinger S. Appropriate finite element formulations for 3D electromagnetic field problems. IEEE Transactions on Magnetics 2002; 38:513-516.

21. Huber JE, Fleck NA, Landis CM, McMeeking RM. A constitutive model for ferroelectric polycrystals. Journal of the Mechanics and Physics of Solids 1999; 47:1663-1697. 

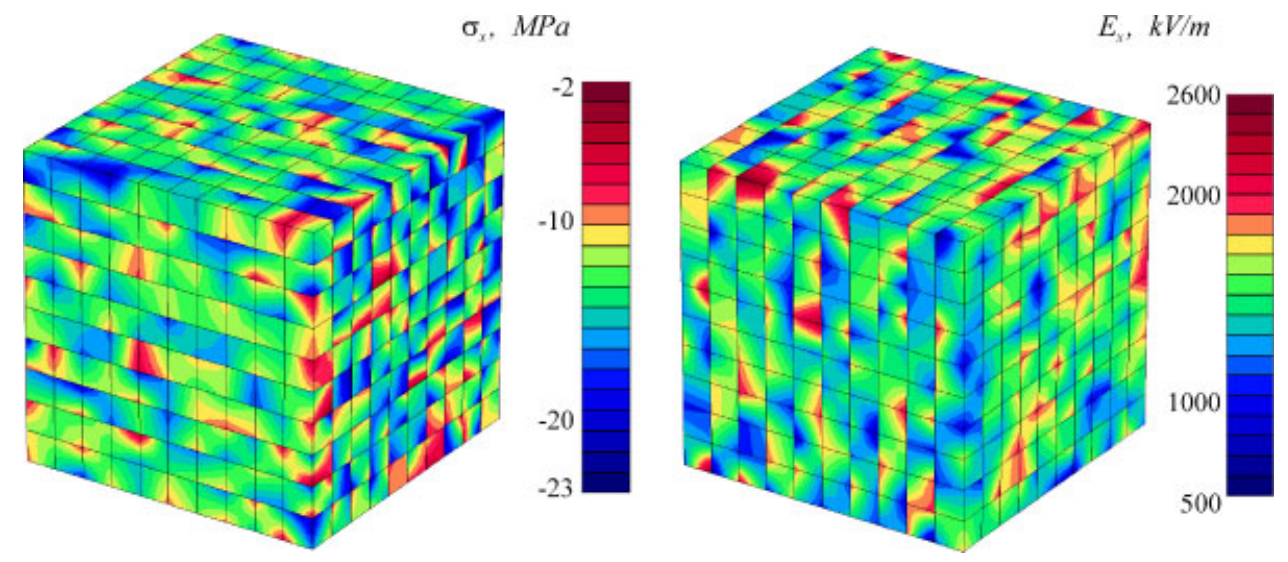

Plate 1. Stress $\sigma_{x}$ and electric intensity $E_{x}$ field distributions in ferroelectric polycrystalline. 